\title{
ALIMENTACIÓN COMPLEMENTARIA EN EL PRIMER AÑO DE VIDA
}

\author{
ANA María ReAlPe MUÑOZ, M.D. ${ }^{1}$, HUMBeRTo ORTIZ RUIZ, M.D. ${ }^{2}$
}

\section{RESUMEN}

La Organización Mundial de la Salud define la alimentación complementaria como "el acto de recibir alimentos sólidos o líquidos (excepto medicamentos en gotas y jarabes) diferentes a la leche, durante el tiempo que el lactante está recibiendo leche materna o fórmula infantil". El primer año es un período de la vida crítico y fundamental, en el que se establecen unos hábitos nutricionales para conseguir el crecimiento adecuado y evitar las enfermedades relacionadas con la alimentación. El inicio de la alimentación complementaria implica un periodo de transición entre los alimentos líquidos y la alimentación del resto de la vida que es principalmente solida.

\section{Palabras claves: Alimentación complementaria, Lactantes}

\section{INTRODUCCIÓN}

La Organización Mundial de la Salud (OMS) define la alimentación complementaria como "el acto de recibir alimentos sólidos o líquidos (excepto medicamentos en gotas y jarabes) diferentes a la leche, durante el tiempo que el lactante está recibiendo leche materna o fórmula infantil",

El concepto de alimentación complementaria como aquello que complementa como una unidad a la lactancia materna sin desplazarla, es relativamente reciente. Surge de la revalorización de la lactancia materna como el alimento optimo para los primeros meses de vida y de la necesidad de diseñar con bases científicas la alimentación para cubrir los requerimientos a partir de los 6 meses $^{2}$.

El término alimentación complementaria comprende un proceso que se inicia cuando se introduce cualquier tipo de alimento no lácteo, como complemento a la leche

${ }^{1}$ Estudiante de posgrado en Pediatría. Universidad del Valle. Cali, Colombia

${ }^{2}$ Pediatra. Profesor. Universidad del Valle. Cali, Colombia

Recibido para publicación: noviembre 15, 2015

Aceptado para publicación: diciembre 15, 2015

\section{SUMMARY}

The World Health Organization defines complementary foods as "the act of receiving solid foods or liquids (except drugs in drops and syrups) other than milk, during the time the infant is receiving breast milk or infant formula." The first year is a period of critical and fundamental life, in which some nutritional habits are established for proper growth and prevent diet-related diseases. The start of complementary feeding involves a period of transition between liquid foods and feeding the rest of life that is mostly solid.

Key words: Complementary foods, Infants

materna o fórmula adaptada (y no como sustitución de ésta), dado diariamente de un modo regular y en cantidades significativas, y termina cuando el niño recibe una alimentación muy parecida a la del resto de la familia ${ }^{3}$.

Estudios en animales han demostrado que las modificaciones en la dieta en etapas tempranas de la vida pueden tener consecuencias metabólicas importantes a largo plazo. Por ello, el primer año es un período de la vida crítico y fundamental, en el que se establecen unos hábitos nutricionales para conseguir el crecimiento adecuado y evitar las enfermedades relacionadas con la alimentación ${ }^{3}$.

El inicio de la alimentación complementaria implica un periodo de transición entre los alimentos líquidos y la alimentación del resto de la vida que es principalmente solida. Esto implica un proceso complejo y que requiere que el niño alcance la madurez necesaria de diferentes órganos y sistemas incluyendo el sistema neuromuscular, renal, digestivo e inmunológico ${ }^{1}$.

Aún en la actualidad no existen bases científicas para ser dogmáticos y establecer normas rígidas en cuanto al porqué, qué, cómo y cuándo deben introducirse los distintos nutrientes. No obstante, ha de tenerse siempre en cuenta los factores nutricionales, geográficos, 
económicos, sociales y culturales, sin olvidar que existe una gran variabilidad individual durante los primeros meses de la vida respecto a las necesidades nutricionales del lactante y circunstancias familiares que le rodean ${ }^{3}$.

Según las recomendaciones actuales de la Academia Americana de Pediatría (AAP) los lactantes estarían preparados para recibir alimentos diferentes a la leche entre las 16 y 27 semanas de vida. Sin embargo se continúa haciendo énfasis en la importancia de la lactancia materna exclusiva durante los primeros 6 meses ${ }^{1}$.

La principal justificación para el inicio de la alimentación complementaria es de tipo nutricional, pues en los primeros 6 meses la leche materna o en su defecto, la leche de formula, es suficiente para permitir el crecimiento del lactante. A partir de esta edad y desde un punto de vista energético, haría falta un gran volumen de leche para cubrir las necesidades que precisa el ritmo de crecimiento y actividad del niño, lo que difícilmente podría ser producido por la madre o lo permitiría la capacidad del estómago del lactante ${ }^{3}$.

El proceso de alimentación del niño puede dividirse en 3 fases según la AAP: lactancia (primeros 6 meses), transición (6-8 meses) y modificada del adulto (8-24 meses $)^{1,3}$.

Se han descrito ciertas "señales" o aspectos que permiten evaluar la madurez de los diferentes sistemas y así poder identificar en qué momento el niño está preparado para iniciar este periodo de transición ${ }^{1}$.

En el sistema neuromuscular señalan que a pesar que desde el nacimiento se cuenta con los reflejos de succión y deglución, la capacidad para deglutir alimentos semisólidos y con cuchara alcanza su madurez entre el 46 mes de vida. Además, el reflejo de extrusión, desaparece hacia los 4 meses. Otro factor importante es el control del cuello y del tronco (sostén cefálico y sedestación) que alcanzan su madurez alrededor del 4 mes. Así entonces, las señales de que un niño está listo para recibir alimentación complementaria incluirían: que pueda mantenerse sentado con poco apoyo, se incline hacia adelante al observar el alimento, muestre interés por él, abre la boca fácilmente al rozar sus labios, no hay reflejo de extrusión, puede desplazar el alimento hacia atrás y deglutirlo ${ }^{1,2,4}$.
Cuando se introducen los semisólidos inicialmente los lactantes chupan o succionan los alimentos de la cuchara. A los 6 meses aparecen movimientos de ascenso y descenso de la mandíbula, tipo masticatorios, que permiten el consumo de alimentos sólidos como galletas o cereales independientemente de la presencia de dientes. Los movimientos laterales de la lengua que llevan los alimentos hacia los molares aparecen a los 810 meses, mientras que los movimientos rotatorios completos que permiten masticar la carne aparecen entre los 12-18 meses. Estos eventos nos explican la edad mínima a la que los alimentos pueden ser manejados físicamente por el niño. La fuerza de la masticación y con ello la eficiencia para cortar, aplastar y triturar alimentos aumenta con la edad. La eficiencia masticatoria a los 6 meses es del $40 \%$ la del adulto y es completa solo a los 16 años ${ }^{2}$.

En el sistema gastrointestinal, entre los 4-6 meses el intestino tiene el desarrollo suficiente para funcionar como "barrera" y evitar la entrada de alérgenos alimentarios. Ya están disponibles en cantidad y calidad las diferentes enzimas para la digestión, evitando intolerancias alimentarias ${ }^{1}$.

La capacidad del estomago es de $10-20 \mathrm{cc} / \mathrm{kg}$ en el recién nacido y va aumentando hasta alcanzar $300 \mathrm{ml}$ al final del primer año de vida. La mayoría de los lactantes presentan un patrón bifásico de evacuación gástrica con un periodo rápido de 20 minutos. Los alimentos pueden influir en el vaciamiento gástrico. La mayor osmolaridad, alta densidad calórica retardan el vaciamiento y los líquidos tienen un vaciamiento mas rápido ${ }^{2}$.

Existe evidencia que hasta los 6 meses de edad la amilasa pancrática es insuficiente por lo que dar almidón antes de esa edad puede producir diarrea. La lipasa es escasa al nacer, se duplica al mes pero permanece baja durante el primer año de vida, los ácidos biliares intraluminales están por debajo del nivel miscelar critico lo que condiciona algún grado de mal absorción grasa. A partir de los 6 meses la absorción de grasa es adecuada (90-95\%) ya que la lipasa pancreática ha alcanzado niveles adecuados y la secreción de sales biliares está completamente desarrollada ${ }^{2}$.

La absorción de antígenos a través de la mucosa intestinal está limitada o inhibida por componentes 
inmunológicos y no inmunológicos, estos últimos incluyen el peristaltismo, saliva, acidez gástrica, flora intestinal y proteasas que contribuyen a la degradación de antígenos. Entre los inmunológicos se encuentran el tejido linfoide asociado a mucosas y la presencia de inmunocomplejos en el moco ${ }^{2}$.

Desde el punto de vista renal, cerca a los 6 meses se alcanza la funcionalidad necesaria para manejar una carga de solutos elevada, alcanzando el $75-80 \%$ de la función del adulto hacia los 12 meses $^{1}$.

Dado que la capacidad máxima de concentración renal no se alcanza hasta el segundo semestre, el niño pequeño tiene dificultades para manejar la sobrecarga de solutos, especialmente en condiciones de baja ingesta de líquidos o de perdidas excesivas ${ }^{2}$.

También debe tenerse en cuenta que es importante no retrasar el inicio de la alimentación complementaria pues los requerimientos nutricionales cambian y ya la leche no aporta los suficientes nutrientes para el adecuado crecimiento y desarrollo del niño. A partir del 6 mes el niño necesita mayor aporte energético, en especial ciertos nutrientes como hierro, zinc, calcio, y las vitaminas $\mathrm{A}, \mathrm{C}$ y D ${ }^{1,2}$.

La introducción de la alimentación complementaria debe iniciarse siempre en pequeñas cantidades, realizando los cambios, ya sean cuantitativos o cualitativos, de forma individualizada, lenta y progresivamente ${ }^{3}$.

Hacia los 6 meses, debe iniciarse con alimentos frescos, higiénicos, suaves, fáciles de digerir, sin demasiada consistencia o que contengan partes sólidas que puedan atorar al bebé. Se aconseja iniciar con compotas de frutas, papillas vegetales o de cereales. Hacia los 7 meses, se pueden empezar a combinar alimentos, aumentar progresivamente la consistencia, ampliar las opciones de alimentos, carnes, purés, compotas y coladas. A los 8 meses ya han desarrollado el movimiento de pinza (dedo pulgar e índice) y por lo tanto se recomienda introducir "trozos" de alimentos que puedan agarrar con la mano (frutas, pan, galletas). Esto promueve el avance de la dentición, masticación y coordinación ente boca-mano. Al año el bebe debe estar ingiriendo la dieta familiar, manejando esquemas y horarios de los adultos: 3 comidas principales y 1-2 refrigerios. Como en todo período de la vida, la alimentación complementaria también debe cumplir con las leyes de Escudero, CESA (completa, equilibrada, suficiente y adecuada) ${ }^{1,2,4}$.

Existen riesgos asociados a la introducción demasiado temprana o muy tardía de la alimentación complementaria y se resumen a continuación:

\section{Riesgos de alimentación complementaria precoz (antes del 4 mes):} principal $^{1,4}$

Se desplaza la leche materna como alimento

- $\quad$ Aumento de enfermedades respiratorias ${ }^{1}$

- Daño renal por sobrecarga de nutrientes (proteínas y minerales) $^{1,2,4}$

Desnutrición ${ }^{1,4}$

- Anemia y deficiencias nutricionales específicas por interferencias en la absorción de nutrientes presentes en la leche materna o por disminución en la ingesta de leche ${ }^{1}$

Alergias ${ }^{1,6}$

Aumento de riesgo de desarrollar hipertensión, obesidad, diabetes, enfermedades intestinales ${ }^{1}$

\section{Riesgos de introducción tardía (después del 6-7 mes):}

Deficiencias nutricionales específicas (hierro, Vitamina A, zinc) ${ }^{1}$

- Crecimiento lento, alteración de la longitud ${ }^{1,4}$

- Desnutrición ${ }^{1-4}$

- Trastornos alimentarios: vómitos, rechazo a los sólidos, alteraciones en la deglución, atoramiento por sólidos
Trastornos del lenguaje ${ }^{1}$
Se ha encontrado que la introducción tardía de alimentos sólidos se ha asociado con aumento del riesgo de sensibilización alérgica a alimentos y alérgenos inhalados ${ }^{6}$
En cuanto a la cantidad de alimentos que debe consumir el niño en el día, se recomienda que a mayor edad el niño debe consumir mayor número de alimentos sólidos e ir disminuyendo las tomas de leche. Según la OMS el número de comidas diarias recomendadas de acuerdo a la edad son ${ }^{2}$ :
- Menor de 6 meses: lactancia materna a demanda
6 meses: lactancia materna +1 comida
7-8 meses: leche materna +2 comidas 
9-12 meses: leche materna +3 comidas

Segundo año: leche materna +4 comidas

La forma habitual de introducir la alimentación complementaria es ir sustituyendo, de una en una, las tomas de leche que recibe el lactante separando la introducción de un alimento nuevo de otro, al menos una semana, para que el niño vaya aceptando los nuevos componentes de la dieta, dando tiempo a la adaptación de su organismo, y pueda detectarse más fácilmente qué alimento específico es el causante de los eventuales problemas que puedan presentarse (alergia o intolerancia) $)^{3}$.

La frecuencia de la exposición a los diferentes gustos varía la aceptación. La preferencia por ciertas comidas o alimentos aumenta con la exposición repetida, por lo tanto se aconseja introducir los nuevos alimentos de a uno por vez y en forma y de manera reiterada hasta que sea aceptado ${ }^{2}$.

La elección de los alimentos depende de factores geográficos, hábitos culturales, disponibilidad, costumbres familiares y nivel socioeconómico de la familia, entre otros ${ }^{1}$.

Según la OMS los Principios de orientación para la alimentación complementaria del niño amamantado, establecen estándares para el desarrollo de recomendaciones locales sobre la alimentación. Estos principios brindan una guía sobre los comportamientos deseables, relacionados con la alimentación, así como recomendaciones sobre la cantidad, consistencia, frecuencia, densidad energética y contenido de nutrientes de los alimentos. Los 10 principios son ${ }^{4}$ :

1. Practicar la lactancia materna exclusiva desde el nacimiento hasta los 6 meses de edad, introducir los alimentos complementarios a partir de los 6 meses (180 días) y continuar con la lactancia materna

2. Continuar con la lactancia materna frecuente y a demanda hasta los 2 años de edad o más

3. Practicar la alimentación perceptiva, aplicando los principios de cuidado psico-social

4. Ejercer buenas prácticas de higiene y manejo de los alimentos

5. Comenzar a los seis meses de edad con cantidades pequeñas de alimentos y aumentar la cantidad conforme crece el niño, mientras se mantiene la lactancia materna
6. Aumentar la consistencia y la variedad de los alimentos gradualmente conforme crece el niño, adaptándose a los requisitos y habilidades de los niños

7. Aumentar el número de veces que el niño consume los alimentos complementarios conforme va creciendo

8. Dar una variedad de alimentos ricos en nutrientes para asegurarse de cubrir las necesidades nutricionales

9. Utilizar alimentos complementarios fortificados o suplementos de vitaminas y minerales para los lactantes de acuerdo a sus necesidades

10. Aumentar la ingesta de líquidos durante las enfermedades incluyendo leche materna (lactancia más frecuente), y alentar al niño a comer alimentos suaves, variados, apetecedores y que sean sus favoritos

No hay argumentos científicos sólidos que demuestren ventajas sobre el orden de introducción de los diferentes alimentos (harinas, verduras o frutas); por lo que, se atenderá a los hábitos y costumbres, y evitando la monotonía ${ }^{3}$.

A continuación un resumen de las recomendaciones acerca de la introducción de los diferentes tipos de $\operatorname{alimentos}^{1-4}$ :

1. Frutas: la AAP recomienda las frutas (en compota) como alimentos semisólidos de primera línea. No dar en el primer año fresas o kiwi (frutas acidas) que se consideran con mayor riesgo de alergia. Idealmente las frutas deben administrarse en forma de papilla, con menos agua, pues así se asegura un mayor aporte energético

2. Verduras: iniciar entre el 5-6 mes con sopa de verduras o puré de verduras, evitar las de hoja larga (espinacas, acelgas, col, remolacha, espárragos, nabos) por mayor riesgo a intolerancia. (gases)

3. Cereales: la AAP recomienda los cereales infantiles enriquecidos con hierro como alimentos semisólidos de primera línea. Iniciar con cereales de arroz y maíz y posteriormente introducir aquellos que contienen gluten

4. Carnes: iniciar entre los 6 meses y medio y 7 meses, preferentemente carne roja ya que su aporte de hierro es mayor

5. Gluten: trigo, avena, cebada y centeno entre el 4-7 mes de vida, de forma gradual $y$ 
acompañarlo de leche materna. Al parecer la leche materna protege contra el desarrollo de enfermedades autoinmunes, como diabetes mellitus tipo 1 y enfermedad celíaca. No debe introducirse ni antes de los cuatro meses ni posterior a los siete, ya que la introducción precoz o tardía han demostrado el aumento en la susceptibilidad o en la incidencia de estas enfermedades

6. Legumbres: Se recomienda iniciar su aporte a partir del $10^{\circ}$ ó $11^{\circ}$ mes debido a su alto contenido en nitratos, siempre añadidas a las verduras, con lo que se enriquece su valor biológico proteico. Su administración será muy gradual y progresiva, 1 ó 2 veces por semana, favoreciendo así el incremento de la actividad enzimática y digestiva y evitando la flatulencia

7. Jugos: la AAP recomienda que hasta los 6 años de vida el consumo de jugos debe ser de máximo 6 onzas al día. Cantidades mayores se asocian con aumento en la ingesta energética, desplazamiento de la leche o de alimentos nutritivos, disminución en la ingesta de vitaminas y minerales, particularmente aquellos vinculados con la salud ósea (calcio y vitamina D), mal absorción, diarrea crónica, alteración de la talla, y predisposición a obesidad

8. Leche de vaca: no antes del primer año de vida por su impacto negativo sobre el estado nutricional de hierro en el lactante entre otros

9. Alimentos alergénicos: se carece de evidencia suficiente y convincente que valide la necesidad y las ventajas de restringir alimentos con potencial alergénico, tales como huevo, pescado, trigo, durante el primer año de vida en niños sanos o en los que tengan antecedentes familiares de alergias, como mecanismo de protección para el desarrollo de estas

10. Huevo: Los datos sugieren fuertemente que introducir huevo cocinado entre los 4-6 meses de edad pueden proteger contra la alergia al huevo y que retrasar su introducción 10-12 meses podría exacerbarla

11. Hierro y zinc: el $90 \%$ del hierro y el $90 \%$ del zinc que requiere el lactante se deben cubrir con los alimentos sólidos

12. Sal y azúcar: evitar durante el primer año de vida

13. Miel: evitar en el primer año de vida, riesgo de contaminación con toxina botulínica

14. Alimentos "peligrosos": nueces, maní, maíz pira y uvas pasas, entre otros, son alimentos peligrosos para los niños por debajo de los cuatro años de edad, dado el riesgo de atoramiento, bronco aspiración, o la probabilidad de que se introduzcan por curiosidad en las fosas nasales u oídos

\section{REFERENCIAS}

1. Daza W, Dadán S. Alimentación complementaria en el primer año de vida. Precop SCP. Volumen 8 Número 4

2. Sociedad Argentina de Pediatría. Guía de alimentación para niños sanos de 0 a 2 años. Primera edición. Argentina 2001

3. Coronel C, Cinta M. La alimentación complementaria en el lactante. Pediatría Integral 2007; XI: 331-344

4. Organización mundial de la salud. La alimentación del lactante y del niño pequeño, capitulo modelo para libros de texto dirigidos a estudiantes de medicina y otras ciencias de la salud. Washington, D.C. 2010

5. Koplin J, Osborne N, Wake M and cols. Can early introduction of egg prevent egg allergy in infants? A population-based study. American Academy of Allergy, Asthma \& Immunology 2010; 126 (4)

6. Nwaru B. Age at the Introduction of Solid Foods During the First Year and Allergic Sensitization at Age 5 Years. Pediatrics 2010; 125: (1) 